in Brussels, Belgium. The 7th Congress will be in 2019, which will provide possibility of knowledge and experience sharing among international delegates.

Conclusion FOHNEU has grown and changed over the past 25 years. This work give an overview about FOHNEU's history and development since the constitution. FOHNEU look forward to the continuing and developing collaborative projects through the strong links created with new and existing memberships.

\section{Occupational Health in the Chemical Industry}

\section{IMPLEMENTING GOOD PRACTICES FOR THE SAMPLING OF CHEMICALS: THE ALTREX SOFTWARE}

Frédéric Clerc*, Nicolas Bertrand, Gautier Mater. INRS, 65 boulevard Richard Lenoir, 75011 Paris, France

\subsection{6/oemed-2018-ICOHabstracts. 1022}

Introduction During their work shift, workers may be exposed to chemicals. In France, when a risk is identified to a workplace, atmospheric concentrations must be measured to determine worker's exposure.

Methods The regulatory authorities in France have published a list of Occupational Exposure Limits Values (OELV) concerning 143 substances and guidelines to establish the sampling strategy, including statistical methods to determine when exposure exceeds its OELV. This approach is similar to the one that will soon be published in the European standard EN689. Result The good practices for the sampling strategy promoted by INRS begin with a comprehensive chemical risk assessment. The similar exposure groups and the number of samples to be collected have to be determined, as well as the organisation of the sampling in the company: this is the sampling strategy. Afterwards, the statistical assessment can be performed if enough data is collected. In this situation, several computing methods can be applied, these are implemented in the open source Expostats library. To assist hygienists in designing strategies and assessing exposure, INRS launches a new version of Altrex, a web-based software that use Expostats.

Discussion The old version of Altrex required important update to conform to current good practices promoted by INRS. The good practices proposed and the assistance provided with Altrex can help industrial hygienists in performing a correct risk assessment and thus implementing appropriate risk management measures.

\section{OCCUPATIONAL EXPOSURE TO CARBON NANOTUBES: THE STATE OF KNOWLEDGE}

Guseva Canu Irina*. Institute for Work and Health, University of Lausanne, Lausanne, Switzerland

\subsection{6/oemed-2018-ICOHabstracts. 1023}

Introduction In an emerging field of nanotechnologies, assessment of exposure is an integral component of occupational and environmental epidemiology, risk assessment and management, as well as regulatory actions. This review focuses occupational exposure to carbon nanotubes (CNT).
Methods PubMed and Scopus databases were searched for period 2000-2017 using all keywords combinations based on the following structure: 'assessment' and 'exposure' and 'carbon nanotube'. The words 'assessment' and 'exposure' were alternatively replaced by 'measurement' and by 'human' and 'occupational', respectively. The word 'carbon nanotube' was alternatively replaced by 'single-walled carbon nanotube', 'double-walled carbon nanotube', 'multi-walled carbon nanotube', and their abbreviations. Only field-studies conducted in occupational settings were included. The quality of the exposure measurement protocol and results reporting were reviewed. The results were compared with the current NIOSH recommended exposure limit (REL) of $1 \mu \mathrm{g} / \mathrm{m}^{3}$ respirable elemental carbon (EC) mass-concentration as an 8 hour time-weighted average.

Result Twenty-five studies conducted in R and D laboratories, small-scale pilot-production facilities, and, more rarely, largescale primary or secondary manufacturer/user facilities in the USA (eleven), the Republic of Korea (four), Japan (four), Russia (one) and Europe (four) were reviewed. Open handling of CNT powder during the sieving, mechanical work-up, packaging, and clean-up work-tasks was classified at highest likelihood of exposure. Fourteen most recent studies measured EC concentration, although using different methods and aerosol fractions. All but one studies observed EC values exceeding the REL. The quantification of CNT agglomerates and/or CNT-contained particles lacks methodological standardisation and precluded any comparison of results.

Discussion Currently available occupational-exposure data are limited, because production and use of CNT are relatively recent and workforce sizes remain small. Due to high variability of methods and instruments used for exposure sampling and analysis and of criteria used for interpreting their results, results are difficult to compare. Further effort of methodological standardisation is warranted.

\section{PERFORMANCE OF MODELS TO ESTIMATE OCCUPATIONAL EXPOSURE}

${ }^{1} \mathrm{M}$ van Tongeren* ${ }^{2}{ }^{2} \mathrm{~J}$ Lamb. ${ }^{1}$ Centre for Occupational and Environmental Health, Centre for Epidemiology, School of Health Sciences, University of Manchester, UK; ${ }^{2}$ The Royal Zoological Society of Scotland RZSS Edinburgh Zoo, UK

\subsection{6/oemed-2018-ICOHabstracts. 1024}

Introduction Increasingly, models and tools are used by occupational hygienists, risk assessors and risk managers for estimating exposure to and risk from chemical agents in the workplace. These range from simple, screening tools (tier 1 tools) that provide conservative exposure estimate, to more complex higher tier exposure models. In Europe, tools such as ECETOC TRA, STOFFENMANAGER and ART have been developed and are used predominantly for risk assessment as part of the REACH regulations. Furthermore, tools for estimating exposure to nano-sized particles have been developed (e.g. NANOSAFER, Stoffenmanager-NANO). In recent years, several studies have been carried out to test the performance of these tools.

Methods Several studies were carried out to determine the performance of the exposure tools for chemical agents and nano-materials, including comparison with measurement data as well as inter-user comparisons.

Results The comparison of tool estimates with measurement data suggested that the tier 1 tools appear to be conservative, although 